\title{
Multilinguales
}

\section{Syntaxe des possessions inaliénable et aliénable : approche métaopérationnelle}

Syntax of inalienable and alienable possessions : a metaoperational approach

Jean-François Kpli

\section{(2) OpenEdition}

1 Journals

\section{Édition électronique}

URL : http://journals.openedition.org/multilinguales/1651

DOI : 10.4000/multilinguales. 1651

ISSN : 2335-1853

\section{Éditeur}

Université Abderrahmane Mira - Bejaia

\section{Édition imprimée}

Date de publication : 1 juin 2014

Pagination : 171-184

ISSN : 2335-1535

\section{Référence électronique}

Jean-François Kpli, « Syntaxe des possessions inaliénable et aliénable : approche métaopérationnelle », Multilinguales [En ligne], 3 | 2014, mis en ligne le 03 juin 2014, consulté le 17 septembre 2019. URL http://journals.openedition.org/multilinguales/1651; DOI : 10.4000/multilinguales.1651

Ce document a été généré automatiquement le 17 septembre 2019.

\section{(i) $\odot$

Multilinguales est mise à disposition selon les termes de la Licence Creative Commons Attribution Pas d'Utilisation Commerciale - Pas de Modification 4.0 International 


\title{
Syntaxe des possessions inaliénable et aliénable : approche métaopérationnelle
}

\author{
Syntax of inalienable and alienable possessions : a metaoperational approach
}

Jean-François Kpli

1 L'idée que " les énoncés réfléchissent l'activité structurante de l'énonciateur et que la surface peut présenter des traces visibles des opérations sous-jacentes " (Adamczewski, 1982:5) induit un changement de perspective notable dans l'analyse du discours. Les énoncés portent les traces de l'énonciateur et le linéaire syntaxique est métalinguistiquement motivé puisqu'il comporte des unités qui renseignent sur l'activité de production. Que se passe-t-il alors lorsque l'énonciateur lui-même est directement concerné par l'activité de production syntaxique, notamment lorsqu'il doit structurer des énoncés portant sur les parties de son corps?

2 L'intérêt accordé à la relation d'appartenance appelée possession inaliénable remonte au moins à Lévy-Bruhl (1914) qui avait découvert que les langues mélanésiennes avaient une syntaxe différente pour exprimer les parties du corps par rapport aux autres éléments extérieurs au corps humain. Plus tard, Bally Charles (1926) a introduit la notion de sphère personnelle en élargissant l'aliénabilité aux relations de parenté. Depuis lors, de nombreuses études montrent que les langues du monde, des langues romanes (Hanon Suzanne, 1988) aux langues océaniques (sous-groupe des langues malayo-polynésiennes) (Lichtenberk Frank, 2003), y compris les langues africaines (Creissels Denis, 2009), ont une manière particulière de structurer leur syntaxe des parties du corps et des relations de parenté.

3 La présente étude vise à proposer un regard novateur sur la relation de l'énonciateur aux parties de son corps à la lumière de la grammaire métaopérationnelle telle que prônée par Adamczewski Henri (1983), Delmas Claude (1987), Jean Pierre Gabilan (1992) et l'ensemble des Amis du Crelingua ${ }^{1}$. Nous tenterons dans un premier temps de montrer que dans la dynamique langue-discours, le choix initial des "notions", des programmes de sens à structurer pour aboutir au discours, une langue comme le baoulé 
${ }^{2}$ (Côte d'Ivoire) impose une contrainte syntaxique dans la désignation des parties du corps comme "notion ». Nous démontrerons ensuite que le français impose également une contrainte dans le choix des opérateurs servant à renseigner sur le statut des parties du corps dans la structuration syntaxique de l'appartenance. Enfin, nous verrons que l'acquis structurel imposé en syntaxe est la résultante d'opérations métalinguistiques et que la possession aliénable se structure inchoativement par rapport à la possession inaliénable.

\section{Les notions de possession inaliénable et de possession aliénable}

4 La notion de possession inaliénable est définie comme une relation d'appartenance dans laquelle l'élément possédé ne peut être séparé du possesseur. Ce caractère inséparable de la relation possesseur-possédé s'exprime en syntaxe de manière particulière. A titre d'exemple, dans l'énoncé en français "J'ai mal à la jambe », c'est l'article «la» qui est choisi plutôt que l'adjectif possessif «ma». La possession aliénable, quant à elle, renvoie à une relation d'appartenance dans laquelle l'élément possédé est séparé du possesseur et entraîne une syntaxe différente de celle produite par l'inaliénabilité et pour lesquelles sont utilisés les adjectifs possessifs comme «mon/ ton/son, etc. » au lieu des articles «le/la/les ». On dit par exemple « il a déchiré mon livre » et non "*il m'a déchiré le livre». La relation par exemple entre un livre et son possesseur est bien différente de la relation entre une partie du corps et son possesseur.

5 La notion d'inaliénabilité s'organise syntaxiquement autour de la notion de sphère personnelle définie par Bally (1926) comme "les choses conçues non comme possédées par l'individu, mais comme faisant partie de son être $»^{3}$. Cette définition indique que la notion peut s'étendre à plusieurs types de relation que l'individu entretient: elle peut s'appliquer à la relation que l'individu entretient avec les parties de son corps, à la relation qu'il entretient avec les autres individus auxquels il est apparenté et à la relation qu'il entretient avec les objets dont il a l'usage de manière permanente, comme l'indique Creissels (2009 : 2). Pour des raisons de commodités pratiques nous limiterons cette étude à la relation de l'énonciateur aux parties de son corps.

6 Dans la relation de l'énonciateur aux parties de son corps, l'inaliénabilité provient de ce qu'il n'est pas possible de déposséder l'énonciateur d'une partie de lui-même. En revanche, dans la relation qu'il entretient avec les objets qui ne font pas partie de lui, l'aliénabilité provient de la possibilité de le déposséder de ces objets. Cette interaction constante entre "l'insécable» et le "sécable» est le fondement de la notion de possession et le lieu d'une syntaxe particulière structurée différemment par les langues du monde.

7 En guise d'exemple, notons les énoncés suivants du français : Possession inaliénable :

(1a) J'ai mal à la jambe

(1b) *J'ai mal à ma jambe

(1c) * Ma jambe me fait mal

Possession aliénable :

(2a) Il a déchiré mon livre

(2b) *Il m'a déchiré le livre 
exprimée à l'aide de l'article «la», et l'article possessif typique « $m a$ » est exclu dans les énoncés (1b) et (1c), même si (1c) est parfaitement admis en français ivoirien. L'utilisation de l'article «la» provient du caractère inaliénable de la relation de possession entre l'énonciateur et la partie de son corps. Toutefois, la nature même de cet article indique que l'inaliénabilité n'est pas une raison suffisante pour justifier son occurrence. Nous aborderons la question plus loin.

9 Quant à l'énoncé (2b), l'inacceptabilité vient du caractère aliénable de la relation de possession entre l'énonciateur et l'objet « livre ». Cela semble bien logique puisque dans cet énoncé, avec la présence du réflexif «me» on s'attend à l'un des éléments qui font partie intégrante du sujet, or « livre » n'en n'est pas un, puisqu'il ne fait pas partie de son être (Forel Claire, 2008 : 183).

Les notions de possession inaliénable et de possession aliénable ainsi définies et illustrées, il convient de remonter à la source de la production discursive pour mieux appréhender ces deux concepts, en prenant notamment la «notion» ou « programme de sens ", comme point de départ de la structuration sémantico-syntaxique.

\section{De la désignabilité des parties du corps comme notion}

Dans la dynamique langue-discours, le terme de «notion» renvoie au contenu sémantique d'un lexème en dehors de tout contexte et qui se situe au niveau «langue ", niveau de représentation mentale appelé puissanciel par Guillaume (1975). Ce chercheur fait en effet la distinction entre le nom en « langue » et son emploi en « discours »:

«La théorie de l'article repose tout entière sur le principe simple de la distinction entre le nom en puissance et le nom en effet. C'est parce que ces deux états nominaux sont sentis différents par l'esprit qu'il y a nécessité d'un signe pour les relier. » (p. 89)

Autrement dit, tout nom dépourvu de contexte est une donnée, une notion en puissance apte à sa mise en discours; la notion ayant été récemment définie comme un "système complexe de représentation structurant des propriétés physico-culturelles d'ordre cognitif », par Gilbert E. (1993 : 66). Pour être exprimée en discours, une «notion» passe par le prisme d'opérations de structuration diverses qui en limitent l'extension avant qu'elle ne prenne un sens particulier articulé sur un contexte de production. La notion peut être désignée ou nommée en dehors de tout contexte et être comprise des locuteurs d'une même langue. Si cette opération de désignation paraît évidente dans beaucoup de langues, elle l'est moins dans certaines langues africaines en ce qui concerne la relation de l'énonciateur aux parties de son corps.

En anglais et en français, par exemple, il est possible de désigner les parties du corps comme des notions -par exemple, «head » / «tête » et «arm » / «bras »- en dehors de tout contexte et renvoyer à l'idée exprimée. Au contraire, une langue comme le baoulé exprime la notion des parties du corps comme déjà structurée, c'est-à-dire liée à son relateur d'appartenance. Par exemple :

Tête bé tí (one's head /some body'shead /their head)

Bras bé sa (one's arm/ some body's arm/their arm)

14 Le baoulé impose une contrainte dans la désignation de l'idée de "tête ", de "tête " humaine en l'occurrence. La notion «tí » ne peut exister seule sans le relateur « bé » qui 
indique l'appartenance au possesseur de "tí », c'est-à-dire de "tête ». En revanche, lorsqu'il faut exprimer l'idée de "tête» non humaine, la "tête» d'un animal par exemple, la notion « $t i$ » doit être précédée du nom de l'animal. Par exemple "alua tí » (chien-tête / tête de chien). La contrainte sémantique et syntaxique imposée par l'utilisation de "bé » est née du caractère intrinsèquement inaliénable de la partie du corps. Cette contrainte n'existe pas quand il faut désigner des notions en dehors des parties du corps (possession aliénable). Par exemple «sol » se dit «assiè » et «arbre », «waka».

Ainsi, en baoulé, la désignation de la partie du corps comme notion est obligatoirement précédée de "bé», qui par ailleurs, est également l'expression du possessif correspondant à « leur »/ « their » :

Leur sol béassiè

Leur arbre béwaka

Le baoulé indique donc que la partie du corps est "partie du corps de quelqu'un». L'opérateur "bé " signale donc le statut pré-structuré, pré-possédé de la partie du corps. C'est un métaopérateur puisqu'il indique qu'au niveau notionnel l'entité corps fait déjà partie d'une relation d'appartenance, c'est-à-dire d'une relation sémanticosyntaxique partie-tout. Delmas et al (1993) définissent ainsi le concept de métaopérateur :

«Quand les mots parlent de la texture du linéaire, de l'agencement des autres mots, on dit qu'ils ont une fonction métalinguistique (naturelle).

« Meta » veut dire « à côté », « après », il s'agit donc de mots qui se trouvent à côté

d'autres mots pour mieux les commenter.» (p. 6)

Dans le cas qui nous concerne, « bé » est à côté de «tí » pour en signifier l'appartenance ou plus spécifiquement la pré-appartenance. La structuration syntaxique commence donc dès le domaine notionnel pour les parties du corps.

\section{Du choix des métaoperateurs de la possession inaliénable}

Les études proposées pour expliquer la présence des articles le/la/les, notamment celles, entre autres, de Hanon, S. (1988) et Cooper, W.R. (2002) ne disent pas explicitement pourquoi ce sont spécifiquement ces articles qui apparaissent dans cette syntaxe des parties du corps. Cooper $(2002: 1)$ se contente de fournir des exemples:

(3a) Il se lava les mains

(3b) *Il lava ses mains

Avec une contrepartie en anglais (Cooper $(2002: 2)$ :

(4a) She patted him on the head

(4b) *she patted him on his head

Des questions fondamentales se posent: pourquoi dans l'exemple (3a), malgré l'utilisation de «les », le nom «mains » s'entend comme appartenant au sujet «il», et pourquoi dans l'exemple (3b), «ses» qui est le possessif par excellence ne pose pas « mains » comme appartenant à « il »? Pourquoi utiliser « les » ou " the »? Quelle est la fonction grammaticale de cet article pour qu'il apparaisse nécessairement dans ce genre de structure syntaxique? 
la présence de «the » dans l'énoncé (4a) s'inscrit dans la relation d'appartenance [him $\mathrm{R}$ head]. Autant qu'il peut l'être, « him » a naturellement le trait sémantique [+humain], et de toute évidence possède une "tête». La relation d'appartenance est donc tautologique, type de relation qu'Adamczewski et Delmas (1982:65) qualifient de " Phase $2 »$. La présence de "the» est parfaitement logique puisqu'il a pour vocation d'indiquer que le nom est déjà «évident ». Nous y reviendrons. Tout comme pour l'énoncé (3a), l'orientation de l'opération va également de la gauche vers la droite, c'est-à-dire de "head » à " him ». Dans l'énoncé (4b) par contre, la présence de " his " signale une relation d'appartenance qu'on pourrait qualifier d'externe, comme si « head » n'était pas déjà une partie de " him ». Autant qu'il marque la possession, « his » introduit ici une relation d'appartenance là où l'existence de cette relation n'est plus à prouver, d'où l'inacceptabilité de cet énoncé. Ce possessif semble dédié à la création d'une relation d'appartenance, de manière inchoative. $\mathrm{Si}$ « his book» est admis comme "évident", "his head" pose problème puisque «his» présuppose en quelque sorte l'existence de "head». A ce stade de l'étude, nous pouvons déjà avancer que les possessifs de l'anglais (my, his / her/ ...) et du français (mon/ton/son/...) portent en eux le trait sémantique pertinent du possesseur [+hum]. De ce point de vue, ils semblent avoir pour vocation d'indiquer une relation de possession aliénable. Ce trait pertinent du possesseur déjà inscrit en eux rend tautologique une relation d'appartenance qui impliquerait une partie du corps du possesseur; d'où le recours à un autre opérateur plus apte à indiquer le statut saturé de la relation nominale, le métaopérateur «le/la».

Depuis l'étude de Guillaume (1975) sur l'article "le", la fonction de cet opérateur est passée de l'expression de simple « défini » à une valeur plus grammaticale qui s'inscrit dans le rapport systémique avec l'unité qui lui est proche en système, à savoir « un ». Pour illustrer cette valeur, citons l'exemple d'Adamczewski (1991: 86) :

(5a) Pour nous, un client, c'est sacré

Multilinguales, 3 | 2014 
(5b) Chez nous le client est roi

$$
\text { paradigmatique. Dans l'énoncé (5b) par contre, il ne s'agit plus de nommer, pas plus }
$$

Dans lénoncé (5a), «client» est nommé inchoativement puisqu'll fait l'objet d'un choix que de choisir un terme dans un paradigme. Selon Adamczewski (1991)

«Client est appréhendé thématiquement, en phase 2 : on a dépassé le stade de la nomination et du choix, et l'on a coupé le $\mathrm{N}$ «client » de tout paradigme et par conséquent de toute complémentarité ou voisinage. C'est un peu comme si l'on avait un déjà-client. » (p.87)

En d'autres termes, "le » signifie le statut déjà acquis du nom. Avec cette valeur métalinguistique, puisqu'il est à côté du nom pour décrire ce statut, on comprend aisément qu'il soit le métaopérateur privilégié dans la syntaxe de la possession inaliénable.

Ainsi, dans l'exemple (1a), la présence de « la » se justifie par le caractère prédéterminé, présupposé et acquis de la relation d'appartenance entre "Je » et " jambe». Dans (1b), L'article «la» dit que cette relation existe déjà, que «jambe » est déjà inscrit comme appartenant au sujet « je ». Dans (1c), le possessif « $m a$ » porte à croire que la partie du corps « jambe » est extérieure à « je » et qu'elle lui est attribuée inchoativement, ce qui est inadmissible.

31 L'énoncé (1c) permet d'étayer cette hypothèse sur la valeur du possessif « $m a$ ». En effet, nommer une partie de son corps comme faisant partie de "soi " est inutile puisqu'elle fait déjà partie de soi. C'est ce qu'indique «ma jambe » dans * Ma jambe me fait mal ». Cette logique du français n'est cependant pas admise dans toutes les langues. Le baoulé admet parfaitement l'énoncé :

Mi djayo mi ya (/ma-jambe-fait-moi-mal / ma jambe me fait mal/ j'ai mal à la jambe).

Pour l'énonciateur baoulé, désigner la partie du corps qui est en cause est tout à fait acceptable puisqu'il s'agit de l'isoler pour faire porter sur elle tout le poids de ce que l'énonciateur veut exprimer, c'est-à-dire la douleur. De ce fait, l'opération relève d'un choix paradigmatique pour signifier «c'est ma jambe qui me fait mal», mais pas «mon bras ", par exemple.

Certes, la présupposition de la préexistence de la relation d'appartenance est toujours exprimée, mais ce n'est plus elle qui est pointée mais la partie du corps paradigmatiquement nommée. Dès lors, on comprend mieux des énoncés tels que « mon pied me fait mal ", "ma tête me fait mal» du français de Côte d'Ivoire, vu que ces expressions sont le calque des langues africaines comme le baoulé.

\section{Les opérateurs « avoir » et « être à » de la possession inaliénable}

Toutes les études sur la possession inaliénable posent le problème de la présence du verbe « avoir ». Selon S. Hanon (1988: 2), une phrase comme :

(6a) Marie a les yeux bleus, introduit une " double prédication à caractère de possession » :

(6b) Marie a des yeux

(6c) Les yeux de Marie sont bleus 
Elle attribue ensuite cette double relation d'inclusion (Marie-yeux /yeux-bleus) à la possession inaliénable.

Cette présentation pose deux problèmes. D'abord celui de l'acceptabilité de (6b) dérivé de (6a). En effet, dans quel contexte un énonciateur peut-il produire (6b) ? Si Marie a le trait sémantique [+humain], cet énoncé n'a pas de raison d'être : tout être humain a des yeux. Ensuite, (6c) est à la limite de l'acceptable, à moins que le contexte ne s'y prête : (6a) est un énoncé correct en français. Tout énoncé étant articulé sur un contexte, (6a) et (6c) sont valides en fonction de leur contexte respectif et, dans ce cas, (6c) ne peut être considéré comme dérivant de (6a). Ce dernier étant un énoncé autonome, son acceptabilité s'inscrit dans l'opération qu'effectue « avoir » dans la mise en relation de [Marie] et [yeux bleus].

Adamczewski et Delmas (1982) définissent ainsi cette opération : « le rôle de avoir/have est d'établir une relation entre un sujet de type locatif ( = lieu, siège) et un objet, l'ensemble de la construction ayant pour but d'énoncer une propriété extrinsèque du sujet grammatical » (p.111).

39 Si la fonction grammaticale invariante d' « avoir » est bien d'établir une relation de type extrinsèque en logeant (« localisant ») le prédicat dans le sujet, on comprend aisément le rôle que joue l'article «les» dans le prédicat contenant une partie du corps. Les "yeux » entrant dans une relation d'appartenance intrinsèque, présupposée, acquise avec le possesseur, en l'occurrence le sujet, le prédicat doit porter la trace de cette présupposition; d'où la présence de «les », trace de cet acquis métalinguistique. Ce pré-requis une fois affirmé, la localisation extrinsèque par " avoir » ne peut se faire que grâce à l'élément informationnel «bleus ». Autrement dit, " avoir » ayant pour rôle d'axer la prédication sur le sujet, la structuration du prédicat contenant les parties du corps, qui implique qu'il y a déjà relation entre le sujet et la partie du corps, génère une trace en surface de cet acquis, en l'occurrence «le/la, les», qui signale que le nom auquel ces opérateurs sont attachés est déjà en relation avec le sujet. En revanche, si la partie du corps est caractérisée par un opérateur comme "un/une » dans cette opération de localisation du prédicat dans le sujet avec «avoir », l'énoncé devient incongru voire inacceptable dans ce contexte (par exemple, *Marie a une dent).

Toutefois, rien n'empêche l'énonciateur d'utiliser « un/une » dans une prédication avec « avoir » pour produire le sens qu'il souhaite. Dans ce cas, le sens produit provient de l'intention sous-jacente de briser la relation intrinsèque pour en faire une relation de type extrinsèque en présentant la partie du corps comme extérieure.

41 Examinons l'exemple suivant affiché sur le portail de Yahoo France le 11/ 01/2014: "Scarlett Johansson a une dent contre la France ${ }^{4}$. La partie «Dent " est introduite inchoativement, comme si elle ne faisait pas partie de Scarlett Johansson, et entre dans une relation axée sur un choix paradigmatique, mettant en exergue la « dent » choisie dans un paradigme. La localisation extrinsèque du prédicat "dent contre la France ", dans le sujet par « avoir » avec ce statut inchoatif de « dent», produit alors l'effet de sens escompté de colère attribué au sujet du fait de la rupture de l'inaliénabilité.

Depuis Benveniste, de nombreux auteurs, Riegel (1984) et Moignet (1975) par exemple, considèrent l'opérateur " être à » comme « avoir » inversé. Or, Adamczewski et Delmas (1982 : 110) considèrent qu'ils n'ont pas la même orientation. Dans :

(7a) La voiture est à Pierre,

(7b) Pierre a une voiture, 

de l'énonciation. Quant à (7b), «avoir» loge "voiture» dans le sujet. L'opérateur « avoir » indique donc une relation asymétrique et « être à » une relation symétrique, représentées de la manière suivante :

Pierre $a$ une voiture

$\leftarrow$

La voiture est à Pierre De ce fait, tout comme pour «avoir» et «être à ", «lè » et «tì indiquent respectivement une relation asymétrique et une relation symétrique :

$\rightarrow$

Kofi lè loto

$\leftarrow$

Kofi i yima-mu bé ti blé

(7c)* les yeux bleus sont à Marie extérieur à l'individu Marie, comme dans l'exemple ci-dessus avec l'élément "voiture ». Or, de manière intrinsèque, Marie a des yeux, il s'agit simplement d'en indiquer la couleur. Cette présupposition impose le verbe "avoir» qui oriente la prédication vers Marie. "Être à " introduit inchoativement "yeux», comme si on l'attribuait pour la première fois à Marie, ce qui est inacceptable, à moins que dans un contexte spécifique plusieurs paires d'yeux soient comparées sans qu'on en connaisse les possesseurs, et qu'on attribue les " yeux-bleus » à Marie. C'est peut-être en raison de ces contraintes sémantique et syntaxique que certaines langues optent pour deux verbes distincts pour exprimer l'aliénabilité et l'inaliénabilité.

Le baoulé, par exemple, utilise le correspondant de « avoir »/ " have » pour exprimer la possession aliénable et le correspondant de « être » pour la possession inaliénable :

Aliénable : (8a) Kofi lèloto /Kofi a voiture/ Kofi a une voiture Inaliénable : (8b) Kofi i yima-mu bé ti blé / Kofi ses yeux - sont bleux.

L'opérateur «lè/avoir » du baoulé semble exclusivement dédié à la structuration de la possession aliénable. Il est impossible de dire :

(8c) *Kofi lèyima blé / Kofi a yeux bleus / Kofi a les yeux bleus.

Par contre, «ti/être » de (8b) est l'opérateur privilégié de la possession inaliénable. (8b) est un énoncé contenant deux séquences [Kofi i yima-mu/ Kofi ses yeux] et [bé ti blé/ils sont bleus]. Dans la première séquence, l'énonciateur pose un préalable, un acquis (Kofi ses yeux). Autrement dit, la relation inaliénable est posée comme prémisse à toute autre opération. Puis la séquence subséquente est tissée avec l'opérateur "ti/être » (sont bleus). Ce préalable nous renvoie à ce que nous disions de la partie du corps comme «notion », à savoir que « toute partie du corps a nécessairement un possesseur ».

L'opérateur «lè/avoir » est exclu puisqu'il aurait noué une relation de type extrinsèque L'opra déjà partie de lui. De fait, (8b) est similaire à (7a) : la partie du corps est étroitement rattachée au sujet Kofi et l'ensemble complexe sujet [Kofi-ses-yeux] est qualifié de [bleus]. L'ensemble sujet est thématique et le prédicat est rhématique au sens d'Adamczewski et Delmas (1982). 
51 Au terme de cette étude, nous retenons que la syntaxe de la possession inaliénable se structure métalinguistiquement, c'est-à-dire que les énoncés portent en eux la trace d'une opération qui indique une préexistence de la relation entre l'énonciateur et la partie du corps qu'ils structurent. Cette préexistence, cet acquis, justifie la présence des articles « le/la, les » dans les énoncés français des parties du corps, puisqu'ils indiquent que les noms auxquels ils sont associés sont acquis, déjà intégrés, déjà connus. Le « déjà-possédé » est donc l'environnement logique de l'émergence de ces métaopérateurs

En amont de la structuration sémantico-syntaxique, une langue comme le baoulé est l'exemple patent de la pré-structuration des parties du corps conçues comme notions. On ne peut désigner la partie du corps que comme partie du corps de quelqu'un; d'où l'émergence obligatoire en surface du métaopérateur "bé». Cette langue possède, en outre, deux unités distinctes qui marquent en surface la possession aliénable et la possession inaliénable.

\section{BIBLIOGRAPHIE}

ADAMCZEWSKI H. et GABILAN, Les clés de la grammaire anglaise, Paris, Armand Colin, Paris, 1992.

ADAMCZEWSKI H., Le français déchiffré, clé du langage et des langues, Armand Colin, Paris, 1991.

ADAMCZEWSKI H., « Pour une grammaire métaopérationnelle de l'anglais », in TREMA, n 8, 1983, pp. 5-16.

BALLY Ch., «L'expression des idées de sphère personnelle et de solidarité dans les langues indoeuropéennes ", in Franz Franhauser \& Jud Jakob (éds), Festchrift Louis Gauchat, Aarau, Sauerlander, 1926, pp. 68-78.

BENVENISTE F., « Être et avoir dans leurs fonctions linguistiques », dans Problèmes de linguistique générale I, 1966, Gallimard, Paris, pp. 187-222.

COOPER ROBERT W., « Inalienable possession in Finnish and English : the Use of Possessive Pronouns/suffixes with Nouns designating Parts of the Body ", in Helsinki English Studies, vol. 2. 2002. Consultable sur le site : http://blogs.helsinki.fi/hes-eng/files/2011/03/ HES_Vol2_Cooper1.pdf Helsinki English Studies, Electronic Journal.

CREISSELS D., Les constructions dites « possessives » étude de linguistique générale et de typologie linguistique, thèse de doctorat d'état, Université de Paris IV, 1979.

CREISSELS D., "Catégorisation et grammaticalisation : la relation génitivale en Afrique », dans Nicolaï, R. (éd.), Leçons d'Afrique (Hommage à Gabriel Manessy), 433-454, Paris, Peeters, 2009. Consultable sur le site : http://www.deniscreissels.fr/public/Creissels-gen.mand.pdf ; ou sur le site : www.deniscreissels.fr (Lundi 13 avril 2009)

DELMAS et al., Faits de langue en anglais, Dunod, Paris, 1993. 
DELMAS C., Structuration abstraite et chaîne linéaire, Paris, CEDEL, 1987. GILBERT É., « La théorie des opérations énonciatives d'Antoine Culioli », dans Les Théories de la Grammaire anglaise en France, Hachette Supérieur, 1993, pp. 63-96.

GUILLAUME G., Le problème de l'article et sa solution dans la langue française, Librairie Nizet, Paris, 1975.

GUILLAUME G., Leçons de linguistique, Québec : Les presses de l'Université Laval/Presses Universitaires de Lille, 1947.

HANON Suzanne, « Qui a quoi ? Réflexions sur la possession inaliénable et le verbe avoir en français », in Revue romane, Bind 23, 2, 1988.

LANGACKER R.W., « Possession and possessive construction », in Taylor J.R. \& MacLaury R. (eds.), Language and the cognitive construal of the world, Berlin, Mouton, 1995, pp. 51-79.

LEVY-BRUHL Lucien, L'expression de la possession dans les langues mélanésiennes, Mémoire de la Société de Linguistique de Paris, 19.2, 1914, pp. 96-104.

LICHTENBERK Frank, « Inalienability and possessum individuation », in Symposium on Linguistic Diversity and Language Theories, Boulder, Mai 2003, University of Auckland.

MOIGNET G., « Incidence et attribut du complément d'objet », in Travaux de linguistique et de littérature, Strasbourg 13, 1975, pp. 253-270.

RIEGEL M., « Pour une redéfinition linguistique des relations dites de "possession" et d'"appartenance" ", in L'Information grammaticale, 23, 1984, pp. 3-7.

SPANOGHE A. M., « La syntaxe de la possession inaliénable en français, en espagnol et en portugais : une présentation », in Travaux de Linguistique (GAND), 28, 1994, pp. 205-207.

\section{NOTES}

1. Les Amis du Crelingua, voir le site : www.crelingua.fr

2. Une langue du groupe Kwa de Côte d'Ivoire dont les locuteurs se trouvent au centre de la Côte d'Ivoire, région dont les principales villes sont Bouaké et Yamoussoukro.

3. Publications du Cercle Ferdinand de Saussure VI, Claire Forel, La Linguistique sociologique de Charles Bally, Librairie Droz S.A, Genève, 2008, p. 183.

4. Affiché sur Yahoo France le 11/01/2014. Scarlett Johansson affirme : «Quand je suis arrivée à Paris, au début, je pensais que tout ce qu'on disait sur la grossièreté et l'impolitesse des Parisiens était faux. Je me disais que les gens ne sont pas grossiers, ils sont merveilleux! Mais ça, c'était avant que je ne m'installe à long terme à Paris. Depuis, les gens ont pensé que, puisque j'avais décidé de rester, ils pouvaient redevenir eux-mêmes, à savoir grossiers ».

\section{RÉSUMÉS}

Cette contribution a pour but de mettre en exergue la structuration particulière que l'énonciateur fait de la syntaxe de la relation d'appartenance dans les langues. Elle vise à montrer 
que les éléments appartenant à la sphère sémantique propre à l'énonciateur, notamment les parties $\mathrm{du}$ corps qui participent de la possession dite inaliénable, se structurent métalinguistiquement et que leurs organisations syntaxiques codent dans le linéaire, de manière spéciale certes, mais logique, les traces de cette inaliénabilité. Elle tente enfin de montrer que les éléments qui ne font pas partie de la sphère personnelle de l'énonciateur et qui participent de la possession dite aliénable se structurent avec des opérateurs inchoativement motivés qui matérialisent une distanciation entre l'énonciateur structurant et les entités extralinguistiques aliénables.

This paper aims to highlight the particular structuring operation that the speaker performs in the syntax of possession in languages. It shows that elements belonging to the semantic sphere specific to the speaker, including body parts involved in inalienable possession, are structured metalinguistically and their syntactic organizations encode in the linear structure, albeit in a special way, but all the same logical, traces of this inalienability. Finally, it shows that elements that are not part of the personal sphere of the speaker and are involved in alienable possession are stuctured paradigmatically with operators materializing a distanciation between the speaker and the alienable extralinguistic entities.

\section{INDEX}

Mots-clés : possession inaliénable, possession aliénable, sphère personnelle, approche métaopérationnelle, notion

Keywords : inalienable possession, alienable possession, personal sphere, metaoperational approach

\section{AUTEUR}

\section{JEAN-FRANÇOIS KPLI}

Université Félix Houphouët Boigny - UFR des Langues, Littératures et Civilisations -Département d'anglais

KPLI Yao Kouadio Jean François, docteur en sciences du langage et traduction, enseigne à l'Université Félix Houphouët Boigny, UFR des Langues Littératures et Civilisation, Département d'anglais - Abidjan - Côte d'Ivoire.

- Publications sélectionnées :

«La problématique du sens en Grammaire métaopérationnelle », online publication of LTML, $\mathrm{N}^{\circ} 1$, Abidjan, Côte d'Ivoire, 2008 : www.ltml.ci

« La Traduction métalinguistique I, online publication of LTML, Abidjan, Côte d'Ivoire : www.ltml.ci

"L'Invariant en linguistique, une quête d'ordre dans le désordre ", online publication of LTML $\mathrm{n}^{\circ}$ 9, october 2013, Abidjan, Côte d'Ivoire : www.ltml.ci

"Lexical Transparency and Opacity in the Translation of the Prefix "sub" : A Metalinguistic Approach ", à paraître en juin 2014. 